Journal of Case Reports 2020;10(2):82-85

\title{
Infantile Tuberculous Osteomyelitis of the Proximal Tibia
}

\author{
Faisal Rahman ${ }^{1}$, Iman Solichin ${ }^{2}$, Nurmansyah Hatta Dwiputra ${ }^{2}$ \\ ${ }^{1}$ Department of Orthopaedic \& Traumatology, Cipto Mangunkusumo General Hospital, Faculty of Medicine Universitas Indonesia, \\ Jakarta, Indonesia; ${ }^{2}$ Department of Orthopaedic \& Traumatology, Spine Consultant, Orthopaedic Purwokerto Hospital, Purwokerto, \\ Indonesia.
}

\section{Corresponding Author:}

Dr. Faisal Rahman

Email: me.faisalrahman@gmail.com

This is an Open Access article distributed under the terms of the Creative Commons Attribution License (creativecommons.org/ licenses/by/3.0).

Received : September 13, 2019

Accepted : April 19,2020

Published : April 25, 2020

\begin{abstract}
Background: The manifestations of tuberculosis osteomyelitis in children non-specific. The only early symptoms are pain and swelling of the involved site. Therefore, the disease is often neglected. Case Report: A 17-month-old boy presented with a four-month history of a painful mass over his left lower leg. X-ray of the left knee showed moderate soft tissue swelling over the medial aspect of the left knee. On physical examination, knee mass was fluctuant, minimally tender and non-pulsatile on palpation and without an associated knee effusion. Histological examination of excised specimen confirmed tuberculous granulomas. Post-operatively the child recovered well and was discharged anti-tubercular drugs for 9 months. Conclusion: Early diagnosis of TB osteomyelitis in children poses a challenge. A high index of suspicion for tuberculosis is needed, and it should be included on the list of differential diagnosis in children with musculoskeletal complaints of swelling and stiffness. The absence of pulmonary TB does not exclude the diagnosis of infantile tuberculous osteomyelitis.
\end{abstract}

Keywords: Child, Granuloma, Osteoarticular Tuberculosis, Osteomyelitis, Pain.

\section{Introduction}

Tuberculosis (TB) of the bone is rare in developed Western countries, but it remains a serious problem in many developing countries. Tuberculosis infection can affect any bone in the body. The spine is the most frequent site of involvement, followed by the hip and knee region. The characteristics of tuberculosis osteomyelitis in children are different from those in adults. The clinical findings are not specific. The only early symptoms are pain and swelling of the involved site. Therefore, the disease is often neglected or diagnosis is delayed.

\section{Case Report}

A 17-month-old boy presented with a four-month history of a painful mass over his left lower leg. The child was previously healthy with normal birth developmental history. All his vaccinations, including $\mathrm{BCG}$, were up to date. There was no history of trauma and family history suggestive of pulmonary tuberculosis (TB). General and systemic examination was normal. The local examination revealed an obvious swelling over the medial aspect of his left proximal tibia with normal overlying skin and no focal temperature change. Antero-posterior (AP) X-ray of the left knee showed moderate soft tissue swelling with no lesion [Fig.1]. The patient was not relieved on one week antibiotics.

On physical examination, knee mass was fluctuant, minimally tender and non-pulsatile on palpation and without an associated knee effusion. The child had abnormal gait and pain on passive range of motion of his left knee. No neurovascular compromise of his left lower limb was noted. The left hip and ankle joints were normal. The leucocyte count was increasing, $16.4 \times 10^{9} / \mathrm{L}$. Antero-posterior X-ray of the left knee showed 
moderate soft tissue swelling over the medial aspect of the left knee, as well as a lytic lesion measuring $0.5 \times 1 \mathrm{~cm}$ on the medial aspect of the proximal tibial metaphysis [Fig.2].

A provisional diagnosis of bone tumor with different diagnosis TB osteomyelitis was made and the child was subsequently taken for excision and biopsy. Intra-operatively a thick-walled abscess cavity containing serous fluid was found in the soft tissues on the medial aspect of the left proximal lower leg [Fig.3]. The fluid was aspirated for microscopy, culture, sensivity and biopsies were taken for histopathological evaluation and postoperative radiograph was taken [Fig.4].

Histological examination confirmed tuberculous granulomas with Langerhans giant cells. Post-operatively the child recovered well and was discharged on anti-TB drugs for another 9 months. The importance of monitoring the child's growth over the next several years was discussed with the parents. After the first month, a dramatic clinical improvement was observed. Radiographs at the two-month showed soft callus and the patient's knee had full range of motion without any complaints [Fig.5].

\section{Discussion}

Tuberculosis of the bones and joints is a rare granulomatous infection caused by Mycobacterium tuberculosis. Tuberculosis still remains a major cause of skeletal infection in developing countries, and extra-pulmonary tuberculosis is more common among children. The Bacillus-Calmette-Guerin vaccination itself may also cause tuberculosis osteomyelitis. The origin of the infection is sometimes unclear, as seen in the current series [1].

TB bacilli reach the proximal tibia by hematogenic spread resulting in a metaphyseal focus. In children younger than one-and-a-half years further spread to the epiphysis occurs through the patent transphyseal vessels from this metaphyseal focus. A concomitant septic arthritis can result following drainage of an epiphyseal abscess into

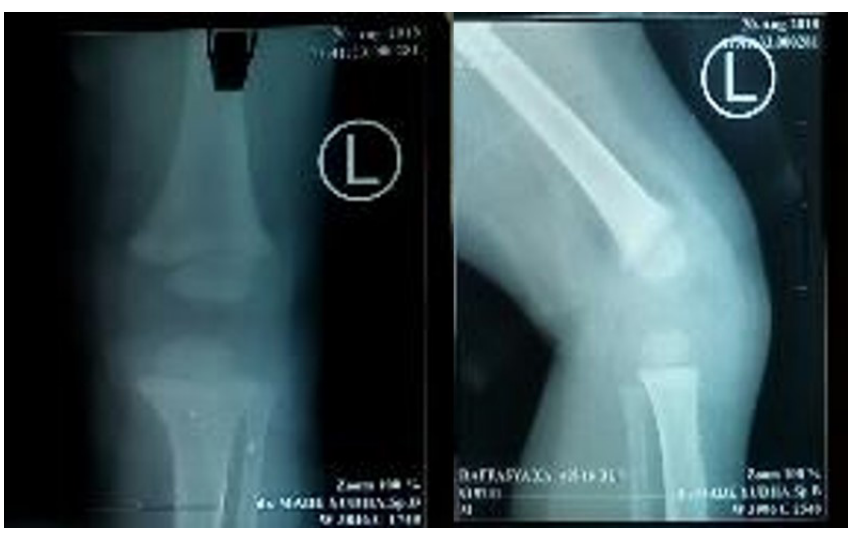

Fig.1: AP and lateral X-ray of the left knee demonstrating soft tissue swelling.

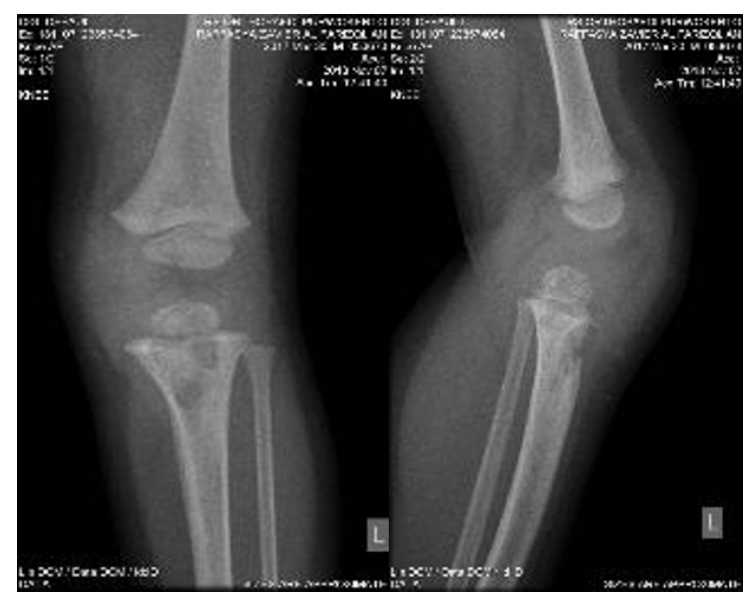

Fig.2: Follow up AP and lateral X-ray of the left knee demonstrating the lytic lesion in the proximal tibial metaphysis.

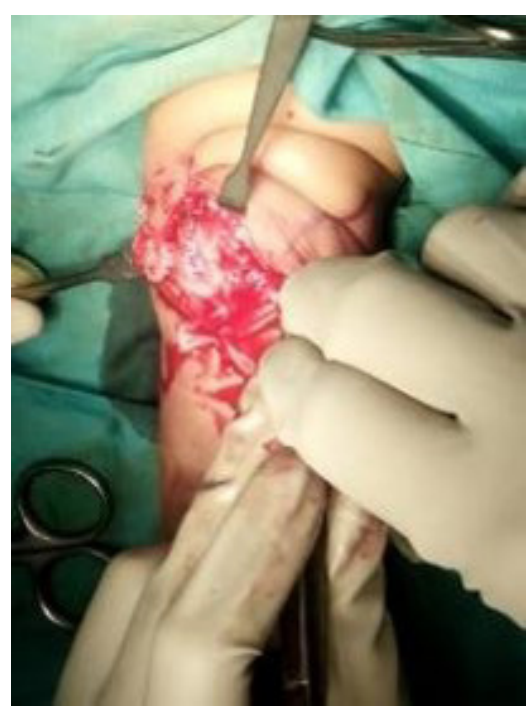

Fig.3: Abscess cavity containing serous fluid. 


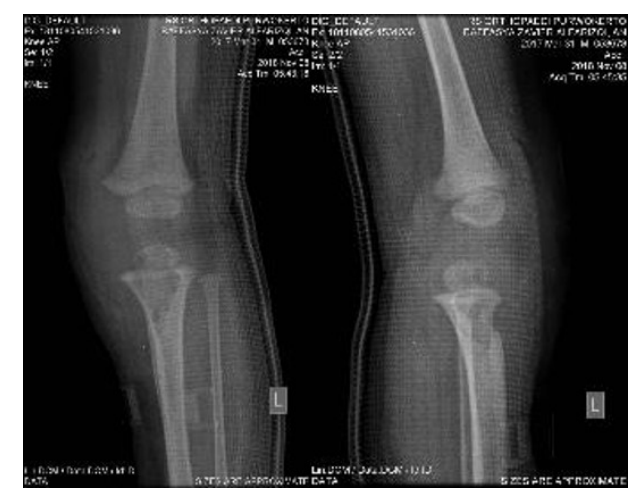

Fig.4: Post-operative radiograph.

the joint through the articular surface, and physeal involvement is explained by direct spread from an adjacent metaphyseal or epiphyseal abscess [2]. The relative rarity of skeletal tuberculosis and nonspecific clinical findings, the diagnosis is usually considerably delayed. Rasool et al. reported the duration of symptoms ranging from 1 to 3 months among 13 children they treated. Primary epiphyseal involvement was seen in only two patients and only two patients had a limitation in the joint movement after treatment [3]. Kerri and Martini reported 98 patients with tuberculosis of the knee; 13 of their patients were children. The average delay was 40 months and one-third of the patients did not have a destructive bony lesion. Teklali et al. reported an average delay in diagnosis of 10 months (range, 10 days to 6 years), indicating the difficulty of making a prompt diagnosis [4,5].

Radiological signs include, but are not limited to: changes in the joint space, sub-chondral erosions, lytic bone lesions and articular osteopenia. Markers of acute infection or inflammation including ESR and CRP are elevated but are non-specific. A definite diagnosis must be made by biopsy and culture. CT and MRI delineated the lesion more precisely and in the planning of subsequent tissue procurement for diagnosis and therapy [6]. The radiological appearance of tuberculosis osteomyelitis may mimic a variety of conditions in children, including pyogenic infections and bone tumors. Malignant processes include the primary pediatric bone cancers (osteosarcoma, Ewing's sarcoma), lymphoma, and

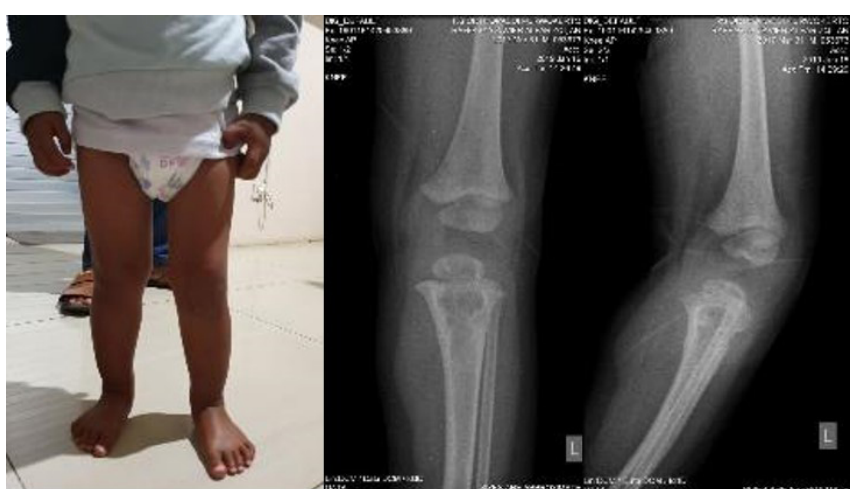

Fig.5: Follow up condition and radiograph.

metastatic lesions from undiagnosed solid tumors elsewhere in the body. Infectious lytic bone lesions in children are most commonly because of gram positive organisms causing osteomyelitis, although gram negative, anaerobic, or atypical organisms may also be found. Even though plain radiographs and CT scan showed typical radiolucent lesions, the MRI showed an aggressive appearance with intra-osseous and extra-osseous extension, and prevented exclusion of pyogenic infections and bone tumors [7].

When performing the biopsy, diagnostic material is most commonly obtained from the destructive area, which reflects the granulomatous focus. If a whitish-gray cheesy material typical of caseous necrosis is seen on macroscopic examination and/or a granulomatous infection can be established (or at least a malignancy is ruled out) on frozen section, then curettage without bone grafting is performed, followed by medical therapy. However, if there is any doubt in terms of diagnosis, then the surgical intervention should be stopped at that point, and the debridement of the granulomatous infection should be performed in the second stage when the diagnosis of tuberculosis is confirmed [8].

The current treatment of skeletal tuberculosis in children includes curettage without bone grafting and anti-tuberculosis drugs. The response to this combined treatment is usually dramatic; significant radiological improvement may occur as early as a few months. Rasool et al. treated 13 children with tuberculosis of bone and 
joints with curettage and anti-tuberculosis treatment [3]. Good clinical and radiological results were obtained, with only two residual joint contractures. In another study, Eren et al. reported five solitary cystic tuberculosis of long tubular bones treated with surgical debridement and chemotherapy. The mean follow-up was 4.3 years, and clinical and radiological healing was observed in all cases [9].

The emergence of drug-resistant organisms has prompted assessment of treatment protocols. Current recommendations for treating active disease require a total of 6 months of drug administration. Most commonly a regimen of isoniazid (INH), rifampin, and pyrazinamide and ethambutol is used. One protocol suggests daily doses of all three drugs for 8 weeks, followed by two- or threetimes weekly doses to a total of 6 months. Another approach is to give daily doses for 2 to 3 weeks, followed by doses twice or three times a week to 6 months. Pyridoxine should be given with INH to prevent peripheral neuritis. Streptomycin is the most commonly used fourth drug. Its main adverse effect is cranial nerve VIII deafness, which is most likely to occur when serum concentrations are too high [10].

Osteomyelitis of the proximal tibia can potentially have devastating long-term complications such as premature joint degeneration and longitudinal growth disturbance. Premature joint degeneration follows epiphyseal destruction while longitudinal growth disturbance is a result of physeal destruction. Song and Kim reported on three cases of non-tuberculous osteomyelitis involving the proximal tibial epiphyses with an eight to 22-year follow-up. They concluded that the epiphysis has remarkable potential for recovery and regeneration and therefore advise expectant management of the bony epiphyseal defects. However, with regard to the physeal destruction, they advise an early osteotomy (before 4 years) to correct angular deformities as recurrence of angular deformity was not seen in their long term followup and the growth plate can recover its potential for symmetrical growth once normal alignment has been restored [11].

\section{Conclusion}

Ahigh index of suspicion for tuberculosis is required in children with musculoskeletal complaints of swelling and stiffness. Furthermore, it should be kept in mind that the absence of pulmonary TB does not exclude the diagnosis of infantile tuberculous osteomyelitis.

Contributors: FR: manuscript writing, literature review and patient management; IS: manuscript editing, and patient management; NHD: critical inputs into the manuscript. FR will act as a study guarantor. All authors approved the final version of this manuscript and are responsible for all aspects of the study.

Funding: None; Competing interests: None stated.

\section{References}

1. Erol B, Topkar MO, Basar H, Caliskan E, Okay E. Solitary cystic tuberculosis of the distal femur and proximal tibia in children. J Pediatr Orthop. 2015;24:315-320.

2. Hiddema WB,Barnard BW, Bouaicha W, Hurter D, Linde GD, Westhuisen CA, et al. Infantile tuberculous osteomyelitis of the proximal tibia involving the growth plate. SA Orthop J. 2012;11:84-87.

3. Rasool MN. Osseous manifestations of tuberculosis in children. J Pediatr Orthop. 2001;21:749-755.

4. Teklali Y, El Alami ZF, El Madhi T, Gourinda H, Miri A. Peripheral osteoarticular tuberculosis in children: 106 Case-reports. Jt Bone Spine. 2003;70:282-286.

5. Kerri O, Martini M. Tuberculosis of the knee. Int Orthop. 1985;9:153-157.

6. Anil A, Neeraj G, Madhusudan M, Nargesh A, Deepak K. Primary epiphyseal and metaepiphyseal tubercular osteomyelitis in children a series of 8 cases. Acta Orthop Belg. 2016;82:797-805.

7. Sehgal K, Fox EJ, Hill RA. Skeletal tuberculosis in children in the Western world: 18 new cases with a review of the literature. J Child Orthop. 2009;3:319-324.

8. Ohtera K, Kura H, Yamashita T, Ohyama N. Long-term follow-up of tuberculosis of the proximal part of the tibia involving the growth plate. J Bone Jt Surg. 2007;399403.

9. Eren A, Fehmi Atay E, Ömeroğlu H, Altintaş F. Solitary cystic tuberculosis of long tubular bones in children. J Pediatr Orthop Part B. 2003;12(1):72-75.

10. Herring JA. Tachdjian's Pediatric Orthopaedics: From the Texas Scottish Rite Hospital for Children. $5^{\text {th }}$ ed. Herring JA, editor. Philadelphia: Elsevier; 2014. pp. 1-1696.

11. Kim HKW, Song KS Regeneration of the proximal tibial epiphysis after infantile osteomyelitis. J Bone Jt Surg. 2005;87:979-983. 